\title{
Is lateral bias anomalous in early-onset schizophrenia? Selected comparisons with normal populations
}

\author{
Simon L. Collinson ${ }^{\mathrm{a}, *}$, Tania Jane Phillips ${ }^{\mathrm{b}}$, Anthony C.D. James ${ }^{\mathrm{a}, \mathrm{c}}$, Digby J. Quested ${ }^{\mathrm{a}}$, \\ Timothy J. Crow ${ }^{\mathrm{d}}$ \\ ${ }^{a}$ Department of Psychiatry, University of Oxford, Warneford Hospital, Oxford OX3 7JX, UK \\ ${ }^{\mathrm{b}}$ CAMHS Academic Unit, Child and Adolescent Mental Health Services, Dunstable Health Centre, Priory Gardens, \\ Dunstable LU6 3SU, UK \\ ${ }^{\mathrm{c}}$ Highfield Adolescent Unit, Warneford Hospital, Oxford OX3 7JX, UK \\ ${ }^{\mathrm{d}}$ Prince of Wales SANE Research Centre, Department of Psychiatry, University of Oxford, Warneford Hospital, Oxford OX3 7JX, \\ $U K$
}

Received 24 March 2003; accepted 28 December 2003

\begin{abstract}
The aim of this study was to investigate lateral bias in patients with early-onset schizophrenia. Hand, eye, and foot preferences and relative hand skill were examined in early-onset patients $(n=44)$ and matched controls $(n=39)$, and were compared with population estimates. Patients demonstrated a significant excess in mixed handedness $(20.5 \%$ vs. $8.5 \%$ ) relative to population estimates and reduced relative hand skill on a pegboard task compared with controls. Left eye preference was significantly less common in schizophrenic patients relative to population estimates. Crossed eye-hand and eye-foot preferences were not significantly increased in the patient group as a whole but were present, respectively, in four of nine and five of nine mixed-handed patients but in none of five mixed-handed controls. These findings are consistent with the view that lateralisation is anomalous in schizophrenia early in the course of illness.

(c) 2004 Elsevier Ireland Ltd. All rights reserved.
\end{abstract}

Keywords: Laterality; Schizophrenia; Handedness; Eye preference; Foot preference

\section{Introduction}

The hypothesis that schizophrenia arises from an underlying defect in cerebral lateralisation has gained support in recent years from studies show-

\footnotetext{
*Corresponding author. Present address: Mental Health Research Institute of Victoria, Locked Bag 11, Parkville 3052, Victoria, Australia. Tel.: +61-3-9388-1633; fax: +61-3-93875061.

E-mail address: scollinson@mhri.edu.au (S.L. Collinson).
}

ing anomalies in the direction and strength of lateral bias (e.g. hand, eye and foot preferences). Review of the literature (Satz and Green, 1999) and meta-analysis (Sommer et al., 2001) concur that schizophrenia is associated with a shift away from strong right-hand preference and an increase in mixed handedness. A smaller literature has shown a reduced prevalence of right-eye preference (Krynicki and Nahas, 1979), excessive left or mixed foot preference (Boklage, 1977; Nasrallah et al., 1982) and anomalies in hand-eye con- 
gruence (Krynicki and Nahas, 1979; Piran et al., 1982).

To date, few studies have investigated lateral bias in early-onset patients. Lishman and McMeekan (1976) reported a shift towards non-right handedness in young patients relative to population norms and controls. Oddy and Lobstein (1972) found ambidextrous schizophrenic patients were significantly younger than right-handed patients. Increased left-eye dominance has been associated with earlier age of hospitalisation (Gureje, 1988) and earlier onset (Oddy and Lobstein, 1972; Krynicki and Nahas, 1979). Anomalous eye-hand congruence has also been associated with earlier onset (Krynicki and Nahas, 1979; Piran et al., 1982). Thus far, only Piran et al. (1982) have examined young patients close to the onset of illness and found greater leftward tendencies on measures of hand, eye and foot preference in early patients compared with neurological patients, psychiatric patients and normal controls.

In this study, indices of hand preference and skill, eye and foot preference, and hand-eye/foot congruence were examined in a sample of earlyonset patients with schizophrenia who were close to first episode. The aim was to determine the presence and character of anomalous lateral preference in relation to both controls and population estimates.

\section{Methods}

Patients ( $n=44,27$ males, 17 females) who met DSM-IV criteria for schizophrenia (American Psychiatric Association, 1994) were recruited from outpatient units in Southern England. The patients had a mean age of 16.7 years (S.D. $=1.3$, age range $=12-20$ ). Most had experienced only one psychotic episode at the time of initial assessment (average duration of illness $=14$ months). In addition, 40 consecutive healthy controls (23 males/ 16 females) were recruited from local general practitioners (mean age $=16.3$, S.D. $=3.0$ ). Patients and controls were closely matched for age and sex ratio, and they had comparable levels of education. One control was excluded because of pre-existing psychopathology. After a complete description of the study, written informed consent was obtained from both the subjects and their parents.

The Annett Hand Preference Questionnaire (AHPQ; Annett, 1970) was administered. The questionnaire consists of 12 questions about hand preference in a range of actions: six 'primary' actions (writing, throwing, hammering, brushing teeth, striking a match, holding a racquet) and six 'secondary' actions (writing, sweeping, shovelling, unscrewing the lid of a jar, dealing cards, threading a needle and holding scissors).

To best capture anomalous preference and to validly compare the results to previous studies, two classifications were applied. The first, "narrow', definition of mixed preference applied to subjects who showed a clear anomalous preference for one of the six primary actions, e.g. five actions are responded to 'right' with one action 'left'). In accordance with Orr et al. (1999), 'either' responses for a primary action were not considered sufficient to warrant classification as mixed handed (Annett groups 5, 6). The second, 'broad', definition differed from the narrow definition in that 'either' hand responses counted as sufficient evidence for a classification of mixed handedness.

Hand skill was assessed with the Annett Pegboard Task. This task involves the placement of 10 pegs within a holed pegboard under timed conditions. To achieve optimal measurement, subjects were encouraged to perform the task as quickly as possible. Each hand was assessed five times. A laterality quotient for relative hand skill was calculated according to the formula: rightleft $/$ right + left $\times 100$.

Eye preference was determined by asking subjects to imagine which eye they would use to look through a telescope. Foot preference was assessed by asking subjects which foot they would use to kick a football into a goal. If subjects were unsure about their preference, they were asked to simulate the action. Subjects were classified as 'right', 'left' or 'either' depending upon preference. These preferences were then compared to the handedness classification to determine hand-eye and hand-foot congruence.

Odds ratio (OR) analyses were conducted comparing early-onset patients and controls and to examine for sex effects. To provide a valid com- 
Table 1

Hand, eye and foot preference in relation to population estimates

\begin{tabular}{|c|c|c|c|c|c|}
\hline Groups & Patients vs. population norm & OR & $95 \% \mathrm{CI}$ & chi-square & $P$-value \\
\hline \multicolumn{6}{|l|}{ Hand preference } \\
\hline$\%$ Mixed handed & 20.5 vs. 8.5 & 2.77 & $1.81-6.52$ & 4.88 & $<0.05$ \\
\hline \multicolumn{6}{|l|}{ Eye preference } \\
\hline$\%$ Left-eyed & 23.0 vs. 37.1 & 0.50 & $0.27-0.93$ & 4.08 & $<0.05$ \\
\hline \multicolumn{6}{|l|}{ Foot preference } \\
\hline$\%$ Left-footed & 11.9 vs. 33.8 & 0.26 & $0.12-0.54$ & 12.6 & $<0.0001$ \\
\hline \multicolumn{6}{|l|}{ Hand-eye } \\
\hline$\%$ Crossed & 28.5 vs. 33.8 & 0.77 & $0.42-0.50$ & 0.44 & ns \\
\hline \multicolumn{6}{|l|}{ Hand-foot } \\
\hline$\%$ Crossed & 19.2 vs. 32 & 0.50 & $0.29-1.09$ & 2.33 & ns \\
\hline
\end{tabular}

Normative samples: (1) Hand preference: Annett (personal communication pending publication). 785 individuals (age 11-17 years) assessed with AHPQ (12 actions); (2) Eye, hand-eye and hand-foot preference: Dargent-Pare et al. (1992), 1530 pooled subjects from questionnaire studies (age $<25$ years).

parison to the normal adolescent population, preferences were also compared with large-scale or meta-analytic normative studies that had used the same or similar assessment methods and classification. For hand preference, schizophrenic estimates were compared with norms provided by Annett (personal communication) taken from 785 healthy adolescents assessed with the AHPQ and classified according to the afore-mentioned criteria. Eye, foot and combined preference estimates were compared with those of 1530 healthy adolescent and young adult right-handed subjects (DargentPare et al., 1992).

\section{Results}

On the basis of the narrow definition, $20.5 \%$ of patients demonstrated mixed handedness compared with $13 \%$ of controls. With the broader definition, $38.6 \%$ of patients and $30.8 \%$ of controls were classified as mixed handed. Neither classification showed a statistically significant difference between patients and controls. There was also no significant excess of mixed hand preference in males or females examined separately. However, when preference rates were compared with normative estimates, the rate of mixed handedness in patients was significantly greater than population estimates with the narrow classification $(20.5 \%$ vs. $8.5 \%$, respectively) $(P<0.05$; see Table 1$)$ and with the broader classification $(38.6 \%$ vs. $17.2 \%$, respectively) ( $\mathrm{OR}=3.20,95 \%$ confidence interval
$\left.[\mathrm{CI}]=1.56-5.83 ; \quad \chi^{2}=10.34, \quad P<0.001\right)$. Within the patient group, there were no significant differences between subjects with mixed or consistent hand preferences with regard to age, age of onset, or illness duration.

To control for leftward bias, left-handed subjects were excluded from the analysis of relative hand skill $(n=5)$. Patients were significantly slower to perform the pegboard task than controls for both the left and right hands (right hand: $F=20.42$, d.f. $=1.72, P<0.001$; left hand: $F=14.89$, d.f. $=$ $1.72, P<0.001)$. When motor slowness was controlled in analysis of covariance (ANCOVA), patients demonstrated a significant reduction in relative hand skill compared with controls $(F=$ 14.03 , d.f. $=1.72, P<0.001)$ and a trend toward a group $\times$ sex interaction $(P=0.07)$ indicating a strong trend for reduced hand skill in male patients relative to male controls, and to females in general.

When non-left-handed subjects were examined, no excess in left eye preference was found in patients compared with controls (12 vs. 9). Compared with population estimates $(28.5 \%$ vs. $37.1 \%$, respectively), however, patients were significantly less likely to show leftward bias. There was no evidence of excess non-right foot preference in patients compared with controls (5 vs. 4 ), although patients were significantly less likely to show left foot preference compared with population estimates $(11.9 \%$ vs. $33.8 \%)(P<0.001)$. The rate of anomalous eye or foot preference was not influenced by the inclusion of sex in the analysis. 
The proportion of crossed preferences for eyehand (nine patients and six controls) and eye-foot (8 and 3) was not significantly different in patients compared with controls. No significant differences were found in the proportion of hand-eye or handfoot consistency between groups when subjects were examined by sex.

\section{Discussion}

We examined indices of hand, eye and foot dominance in early-onset schizophrenia. Previous investigations suggest that anomalous bias may be more common in early-onset schizophrenia, but few studies have directly examined young patients close to the first episode. To our knowledge, this is the youngest sample examined to date. When a narrow definition was used, over $20 \%$ of earlyonset patients demonstrated anomalous hand preference relative to $8.5 \%$ of a normative cohort of healthy adolescents. These findings support the link between mixed handedness and schizophrenia and confirm its presence in young patients early in the course of illness.

The observed rate of mixed preference represents a significant increase from the estimates of adult studies using the AHPQ and current classification. DeLisi et al. (2002) reported mixed handedness in $11.4 \%$ of 275 adult male and $7.8 \%$ of 143 female patients with schizophrenia or schizoaffective disorder. Orr et al. (1999) found $16 \%$ mixed handedness in a sample of 94 schizophrenic subjects. In the only comparable study of young patients, Lishman and McMeekan (1976) found $11 \%$ mixed handedness in psychotic patients (mean age $=25$ years). Several reports indicate higher rates of mixed handedness in adult patients ranging from 36 to $43 \%$ (Green et al., 1989; Nelson et al., 1993; Cannon et al., 1995); however, these studies employed different handedness inventories and applied less restrictive classifications of mixed hand preference. When a similar classification was applied to the current dataset, the rate of mixed handedness in patients corresponded with these estimates.

Although mixed preference was more common in patients compared with controls, the inflated rate of mixed handedness in the control sample prevented statistical significance. Nonetheless, a significant reduction in relative hand skill was observed. Crow et al. (1996), in a cohort study, found that individuals who became psychotic by the age of 28 years were more likely to be reported as ambidextrous for writing hand at age 7 years and were less strongly lateralised on a square checking task than the general population at age 11 years. Others have found altered relative hand skill in adult schizophrenic patients using a similar pegboard task (Sakuma et al., 1996). In view of the correspondence between hand preference and hand skill (Triggs et al., 2000), anomalies detected on these different measures likely represent manifestations of the same underlying disturbance, albeit in differing severity.

Against expectations, we found no significant excess of anomalous eye, foot or eye-hand/foot congruence in this sample. Several studies have failed to find evidence of anomalous eyedness and footedness in adults with schizophrenia (Merrin, 1984; Tiwari and Mandal, 1998), but the present findings conflict with evidence from a cohort study by Cannon et al. (1997) who found that preschizophrenic children had excess left eye dominance at age 11 compared with controls, and with reports of reduced right eye and foot preference in young or early-onset patients (Piran et al., 1982; Gureje, 1988).

It is not entirely clear why leftward eye and foot preferences did not occur in similar excess to the rate of mixed hand preference, but several points warrant consideration, First, although it is assumed that eye, hand and foot preferences are closely related, the strength of association between preferences is moderate; only $65 \%$ of healthy righthanders and $57 \%$ of left-handers are hand-eye congruent depending upon the strength of hand preference (McManus et al., 1999). Second, while hand preference is relatively straightforward in terms of its contralateral organisation within the brain, eye preference, as a consequence of binocular vision and its association with foot and hand preference, is likely to be more complex. Recent studies suggest that eye and foot preference, like hand preference, may be situational and specific to the task at hand (Hart and Gabbard, 1997; Khan and Crawford, 2001). If so, a multi-item assess- 
ment is needed to accurately measure bias. A limitation of the current study is the use of a single item assessment of eye and foot preference.

Finally, it is noteworthy that with the use of a stringent definition of mixed handedness, four of nine mixed-handers showed crossed hand-eye preference and five of nine showed crossed hand-foot preferences, whereas none of the mixed-handed controls were crossed in respect to either eye or foot preferences. Speculatively, this could indicate the existence of a core group of earlier onset patients within the general patient population who are more diffusely lateralised. Such patients may have a greater presence in studies that report reduced bias and an excess of crossed dominance. A study by Seisdedos et al. (1999), which showed earlier clinical onset and smaller brain size in patients with crossed hand-eye dominance relative to uncrossed patients, would appear to support this possibility.

In conclusion, the clear anomaly in hand preference indicates that lateralisation is deviant in early-onset schizophrenia. The degree to which this deviation differs from the older onset schizophrenic population is not yet clear, but the evidence suggests that anomalous hand preference may be more common or possibly more severe in earlyonset patients.

\section{Acknowledgments}

This research project was supported by the Medical Research Council, UK, and SANE. The authors especially thank Prof. Marian Annett as well as Gina Clark, Kristin Bohn, and Mrs Susan James for their contributions.

\section{References}

American Psychiatric Association, 1994. Diagnostic and Statistical Manual of Mental Disorders (DSM-IV). Author, Washington DC.

Annett, M., 1970. A classification of hand preference by association analysis. British Journal of Psychology 61, 303-321.

Boklage, C.E., 1977. Schizophrenia, brain asymmetry development, and twinning: cellular relationship with etiological and possibly prognostic implications. Biological Psychiatry $12,19-35$
Cannon, M., Byrne, M., Cassidy, B., Larkin, C., Horgan, R., Sheppard, N.P., O'Callaghan, E., 1995. Prevalence and correlates of mixed-handedness in schizophrenia. Psychiatry Research 59, 119-125.

Cannon, M., Jones, P., Murray, R.M., Wadsworth, M.E., 1997. Childhood laterality and later risk of schizophrenia in the 1946 British birth cohort. Schizophrenia Research 26, 117-120.

Crow, T.J., Done, D.J., Sacker, A., 1996. Cerebral lateralization is delayed in children who later develop schizophrenia. Schizophrenia Research 22, 181-185.

Dargent-Pare, C., De Agostini, M., Mesbah, M., De, G., 1992. Foot and eye preferences in adults: relationship with handedness, sex and age. Cortex 28, 343-351.

DeLisi, L.E., Svetina, C., Razi, K., Shields, G., Wellman, N., Crow, T.J., 2002. Hand preference and hand skill in families with schizophrenia. Laterality 7, 321-332.

Green, M.F., Satz, P., Smith, C., Nelson, L., 1989. Is there atypical handedness in schizophrenia? Journal of Abnormal Psychology 98, 57-61.

Gureje, O., 1988. Sensorimotor laterality in schizophrenia: which features transcend cultural influences? Acta Psychiatrica Scandinavica 77, 188-193.

Hart, S., Gabbard, C., 1997. Examining stabilising characteristics of footedness. Laterality 2, 17-26.

Khan, A.Z., Crawford, J.D., 2001. Ocular dominance reverses as a function of horizontal gaze angle. Vision Research 41, 1743-1748.

Krynicki, V.E., Nahas, A.D., 1979. Differing lateralized perceptual-motor patterns in schizophrenic and non-psychotic children. Perceptual and Motor Skills 49, 603-610.

Lishman, W.A., McMeekan, E.R., 1976. Hand preference patterns in psychiatric patients. British Journal of Psychiatry 129, 158-166.

McManus, I.C., Porac, C., Bryden, M.P., Boucher, R., 1999. Eye-dominance, writing hand, and throwing hand. Laterality 4, 173-192.

Merrin, E., 1984. Motor and sighting dominance in chronic schizophrenics. Relationship to social competence, age at first admission, and clinical course. British Journal of Psychiatry 145, 401-406.

Nasrallah, H.A., McCalley-Whitters, M., Kuperman, S., 1982. Neurological differences between paranoid and non-paranoid schizophrenia: part I. sensory-motor lateralization. Journal of Clinical Psychiatry 43, 305-306.

Nelson, L.D., Satz, P., Green, M., Cicchetti, D., 1993. Reexamining handedness in schizophrenia: now you see itnow you don't. Journal of Clinical and Experimental Neuropsychology 15, 149-158.

Oddy, H.C., Lobstein, T.J., 1972. Hand and eye dominance in schizophrenia. British Journal of Psychiatry 120, 331-332.

Orr, K.G., Cannon, M., Gilvarry, C.M., Jones, P.B., Murray, R.M., 1999. Schizophrenic patients and their first-degree relatives show an excess of mixed-handedness. Schizophrenia Research 39, 167-176. 
Piran, N., Bigler, E.D., Cohen, D., 1982. Motoric laterality and eye dominance suggest unique pattern of cerebral organization in schizophrenia. Archives of General Psychiatry $39,1006-1010$.

Sakuma, M., Hoff, A.L., DeLisi, L.E., 1996. Functional asymmetries in schizophrenia and their relationship to cognitive performance. Psychiatry Research 65, 1-13.

Satz, P., Green, M.F., 1999. Atypical handedness in schizophrenia: some methodological and theoretical issues. Schizophrenia Bulletin 25, 63-78.

Seisdedos, R.T., Arias, J.S., Gomez, J.S., Beneyto, M., Cercos, C.L., 1999. Early age of onset, brain morphological changes and non-consistent motor asymmetry in schizophrenic patients. Schizophrenia Research 37, 225-231.

Sommer, I., Aleman, A., Ramsey, N., Bouma, A., Kahn, R., 2001. Handedness, language lateralization and anatomical asymmetry in schizophrenia. British Journal of Psychiatry 178, 344-351.

Tiwari, G., Mandal, M.K., 1998. Side bias in schizophrenia: hand, foot, eye and ear. Journal of General Psychology 125, $39-45$.

Triggs, W.J., Calvanio, R., Levine, M., Heaton, R.K., Heilman, K.M., 2000. Predicting hand preference with performance on motor tasks. Cortex 36, 679-689. 